\title{
Les traductions de récits de voyage et leurs arrière-plans politiques
}

\author{
Jan VANDERSMISSEN, \\ Chargé de recherches du Fonds de la Recherche Scientifique-FNRS, \\ Directeur du Centre d'Histoire des Sciences et des Techniques, \\ Université de Liège, Centre d'Histoire des Sciences et des Techniques \\ Extrait de : Michèle COLTELLONI-TRANNOY (dir.), La traduction, sa nécessité, ses ambiguïtés et ses pièges, Paris, \\ Édition électronique du CTHS (Actes des congrès des sociétés historiques et scientifiques), 2015. \\ Cet article a été validé par le comité de lecture des Éditions du CTHS dans le cadre de la publication \\ des actes du $139^{\mathrm{e}}$ Congrès national des sociétés historiques et scientifiques tenu à Nîmes en 2014.
}

Cette contribution s'inscrit dans un projet de recherche financé par le Fonds de la Recherche Scientifique-FNRS qui vise à mettre en rapport l'intérêt accru des savants pour $l^{\prime}$ Afrique à la fin du XVIII ${ }^{\mathrm{e}}$ siècle et l'intensification de la compétition économique et militaire entre la France et la Grande-Bretagne. Ce projet ambitionne de démontrer comment une connaissance de plus en plus précise du terrain africain a influencé la façon dont les gouvernements des deux pays ont intégré l'expertise coloniale dans une politique scientifique complexe appropriée aux besoins spécifiques des deux États ${ }^{1}$.

La notion de "politique scientifique» se réfère à la façon dont un gouvernement applique les connaissances et l'expertise apportées par le monde scientifique en fonction des besoins du pays ${ }^{2}$. En effet, sous l'impulsion de la "nouvelle science », issue de la Révolution scientifique, les administrations coloniales de la France et de la GrandeBretagne se sont tournées vers des connaissances «certaines " reposant sur des observations et des expériences «fiables » faites dans le contexte colonial africain. Si l'Afrique a émergé dans la relation entre le pouvoir et les sciences, c'est surtout grâce aux voyageurs et aux récits qu'ils nous ont laissés, racontant des recherches de terrain et des observations directes effectuées à l'intérieur d'un continent resté inconnu aux Européens pendant plusieurs siècles ${ }^{3}$.

Les récits de voyage ont joué un rôle important dans la circulation des connaissances à l'âge moderne. En Europe, l'exploration de l'Afrique a été suivie de près à la fois par des gens instruits, par des membres de sociétés savantes et par des hommes politiques. Les récits étaient pour eux des sources de connaissances scientifiques, économiques et stratégiques ${ }^{4}$. Des deux côtés de la Manche, les fonctionnaires ont soumis les récits de voyage à des analyses critiques. Ils avaient intérêt à faire traduire les récits produits par la nation concurrente dans leur propre langue. Cette contribution mettra donc l'accent sur la traduction de récits de voyage français et britanniques et sur leurs arrière-plans politiques.

1. Titre complet du projet: L'Afrique dans les "politiques scientifiques " de la France et de la Grande-Bretagne du XVIII siècle au milieu du XIX $X^{e}$ la préparation savante du Grand Partage entre les Puissances.

2. R. Halleux, «Aux origines des politiques scientifiques », p. 439-440.

3. D. Kennedy, The Last Blank Spaces. Exploring Africa and Australia, p. 1-61.

4. J.A. Carney et R.N. Rosomoff, In the Shadow of Slavery. Africa's Botanical Legacy in the Atlantic World, p. 3 ; A.S. Curran, "Imaginer l'Afrique au siècle des Lumières ", Cromohs, p. 1-14 ; A.S. Curran, The Anatomy of Blackness, Science and Slavery in an Age of Enlightenment, p. 29-73 ; C. Gallouët, D. Diop, M. Bocquillon et G. Lahouati (dir.), L'Afrique du Siècle des Lumières : savoirs et représentations, p. xiv ; J. Vandersmissen, «Les voyages organisés par ordre du Roi », p. 491-504. 
Il faut distinguer trois périodes. D'abord, celle de la découverte «scientifique» de l'Afrique au Siècle des Lumières. Ensuite, celle de la prise de pouvoir de l'exploration «banksienne » à la fin du XVIII ${ }^{\mathrm{e}}$ siècle. Enfin, celle de la percée de la science impérialiste dès l'époque napoléonienne. Au cours de chaque période les traducteurs ont joué un rôle non négligeable sur le plan intellectuel et politique, renforçant la diffusion de connaissances « africaines » parmi les lettrés et les puissants partout en Europe.

\section{L'Afrique des Lumières (1720-1788)}

L'exploration de l'Afrique par les Européens a débuté à partir des côtes. Depuis le $\mathrm{XVII}^{\mathrm{e}}$ siècle, les Français, Britanniques, Hollandais, Portugais et Scandinaves se disputaient le contrôle des stations situées principalement dans les régions maritimes de l'Afrique occidentale. Il s'agissait de postes clés pour la traite et pour le commerce de l'or.

Stimulés par le gain ou par la curiosité, certains audacieux présents sur place décidèrent de quitter leur lieu de résidence pour explorer l'arrière-pays. Leurs observations, enregistrées dans des carnets puis envoyées en Europe, permirent les premières descriptions "scientifiques» des zones côtières. Un certain nombre de ces «témoignages» furent publiés sous forme de livres. ${ }^{5}$. Ainsi, les observations d'André Bruë (c.1654-1738), directeur général de la « Compagnie Royale de France aux Côtes du Sénégal et autres lieux d'Afrique", alimentèrent l'ouvrage intitulé Nouvelle relation de $l^{\prime}$ Afrique occidentale, rédigé en 1728 par le polygraphe Jean-Baptiste Labat (1663-1738) ${ }^{6}$. Du côté britannique, l'ouvrage intitulé A New Account of Some Parts of Guinea and the SlaveTrade, de William Snelgrave, commerçant en ivoire et en esclaves, marqua les esprits ${ }^{7}$. Bruë ainsi que Snelgrave expliquèrent à leurs contemporains que l'intérieur de l'Afrique possédait un potentiel économique considérable. Très vite, leurs récits furent mis à la disposition d'un public international à travers des traductions. Publié en anglais en 1734, le livre de Snelgrave reçut une édition française en 1735 grâce à la traduction effectuée par un certain de Coulange ${ }^{8}$.

La diffusion de récits de voyage traduits en plusieurs langues s'accéléra dans le deuxième quart du XVIII ${ }^{\mathrm{e}}$ siècle, grâce à la propagation d'un nouveau genre littéraire : les compilations de récits de voyage. Il s'agit de vastes collections, composées de textes assez hétérogènes, à la fois du point de vue de leur longueur et de leur contenu, mais appartenant tous au genre de la littérature de voyage. Dans la composition de ces collections, qui s'adressent à un large lectorat de personnes cultivées plutôt qu'à des spécialistes, on privilégie les histoires spectaculaires au détriment de réflexions philosophiques ou d'observations factuelles détaillées. L'intérêt commercial l'emporte sur l'argument scientifique. En France, le principal exemple d'une telle collection est l'Histoire générale des voyages, éditée sous la direction de l'abbé Antoine-François Prévost (1697-1763) ${ }^{9}$. La première édition, publiée entre 1746 et 1759 , compte quinze volumes, dont cinq consacrés à l'Afrique. On y retrouve les aventures de Bruë dans la version rédigée par Labat à côté de plusieurs traductions de récits britanniques, dont l'œuvre de Snelgrave. Surtout, cet ouvrage comporte la traduction française du récit de Francis Moore dont l'original, publié en 1738, est intitulé Travels into the Inland Parts of Africa: Containing a Description of the Several Nations for the Space of Six Hundred Miles up the River

5. D. Diop, P. Graille et I. Zatorska (dir.), L'Afrique, p. 1-26.

6. P. Masson, «Une double énigme : André Bruë», p. 9-34 ; P.E.H. Hair, « The Falls of Félou : A Bibliographical Exploration », p. 113-130 ; P. Cultru, «Les faux d'un historien du Sénégal », p. 399-402.

7. R. Law, "The Original Manuscript Version of William Snelgrave's New Account of Some Parts of Guinea », p. 367-372.

8. Nouvelle relation de quelques endroits de Guinée, et du commerce d'esclaves qu'on y fait... Traduite de l'anglois du Capitaine Guillaume Snelgrave, par Mr. A.Fr.D. De Coulange, Amsterdam, Aux dépens de la Compagnie, 1735.

9. J. Sgard, « Prévost : de l'ombre aux lumières (1736-1746)», p. 1479-1487 ; S. Albertan-Coppola, « Des récits des voyageurs à l'Histoire générale des voyages : la représentation des Africaines », p. 165-181. 
Gambia. Ce texte a contribué à la diffusion de nouvelles connaissances géographiques à propos de l'intérieur de l'Afrique en dehors de la Grande Bretagne ${ }^{10}$.

Inversement, des récits français furent intégrés dans des compilations britanniques. Ainsi, la collection intitulée Universal History from the Earliest Account of Time to the Present (1736-1790), contient un certain nombre de récits français traduits en anglais ${ }^{11}$. Il faut néanmoins souligner que ce monument d'érudition a été à son tour traduit et intégralement édité en français par un collectif de commerçants, ce qui démontre la popularité de ce nouveau genre littéraire. L'Histoire universelle depuis le commencement $d u$ Monde jusqu'à présent; composée en Anglois par quelques Gens de Lettres; nouvellement traduite en François par une société de Gens de Lettres, parue dans la seconde moitié du XVIII ${ }^{\mathrm{e}}$ siècle, compte environ cent volumes.

Le $\mathrm{XVIII}^{\mathrm{e}}$ siècle est la période du développement de la recherche "scientifique " sur les côtes africaines. La botanique, considérée comme une science «utile", eut un rôle de précurseur. Nombreux sont les voyageurs-naturalistes qui ont exploré les zones maritimes de l'Afrique à la recherche de graines et de plantes alimentaires ou industrielles : Jean-André Peyssonnel, Thomas Shaw et René-Louiche Desfontaines en Afrique du Nord, Michel Adanson au Sénégal, Francis Masson et François Levaillant au Cap de Bonne-Espérance. En général, ces naturalistes recevaient des instructions, soit d'un puissant souverain ou de son administration, soit d'un riche protecteur, soit d'une société savante. C'est pourquoi ils communiquaient les résultats de leurs recherches aux commanditaires qui pouvaient décider de les garder secrets ou de les rendre publics. Dans ce dernier cas, les informations étaient publiées dans des périodiques ou sous forme de livres ou de mémoires. Ainsi, on trouve des observations d'Adanson ou de Desfontaines dans les Mémoires de l'Académie Royale des Sciences. Les récits circulaient à travers l'Europe grâce aux réseaux établis entre les sociétés savantes ${ }^{12}$. Ainsi, des traductions partielles de ces récits commençaient à circuler elles aussi. La pratique de la traduction était particulièrement employée par la Royal Society : on demandait à des membres experts de rendre les textes écrits en langues étrangères compréhensibles pour leurs confrères. La dissertation sur le corail de Peyssonnel, résultat d'une mission de recherches effectuée en Afrique du Nord, en est l'exemple. Communiqué à la Royal Society en 1751 sous forme d'un long manuscrit, ce texte fut synthétisé et traduit en anglais par William Watson, puis lu en séance, et enfin publié dans les Philosophical Transactions $^{13}$. Inversement, des récits anglais étaient lus et analysés à Paris. On y avait pris l'habitude de publier des traductions commentées dans des périodiques comme le Journal des Sçavans, le Mercure de France, les Mémoires de Trévoux, l'Année littéraire, ou le Journal encyclopédique.

Certains récits de voyage furent publiés intégralement en dépit de leur longueur. On estimait que la valeur scientifique élevée de ces descriptions justifiait des éditions en plusieurs volumes, souvent richement illustrées. Il est étonnant de voir avec quelle

10. M.H. Hill, "Towards a chronology of the publications of Francis Moore's Travels into the inland parts of Africa... ", p. 353-368.

11. G. Ricuperati, « Universal History : storia di un projetto europea. Impostori, storici ed editori nella Ancient Part », p. 7-90 ; G. Abbattista, "The literary mill : per una storia editoriale della Universal History (1736-1765) » p. 91-133 ; Id., " The business of Paternoster Row : towards a publishing history of the 'Universal History', p. 550 ; Id., "The English Universal History : publishing, authorship and historiography in a European project (1736-1790)», p. 103-108 ; H. Inglebert, Le Monde, l'Histoire. Essai sur les histoire universelles, p. 661-662.

12. M.-N. Bourguet, "Voyage, collecte, collections. Le catalogue de la nature (fin XVII ${ }^{\mathrm{e}}$-début XIX ${ }^{\mathrm{e}}$ siècles) ", p. $185-207$.

13. «An Account of a manuscript treatise, presented to the Royal Society, intituled Traite du corail contenant les nouvelles decouvertes, qu' on a fait sur le corail, les pores, madrepores, scharras, litophitons, eponges, et autres corps et productions, pour servir à l'histoire naturelle de la mer; that is to say, A Treatise upon Coral, and Several other Productions furnish'd by the Sea, in order to illustrate the natural History thereof, by the Sieur Peyssonnel, M.D. Correspondent of the Royal Acad. of Sciences of Paris, of that of Montpelier, and of that of Belles Lettres at Marseilles; Physician-Botanist, appointed by His Most Christian Majesty in the Island of Guadelupe, and heretofore sent by the King to the Coasts of Barbary for Discoveries in Natural History. Extracted and translated from the French by Mr. William Watson, F.R.S. », Philosophical Transactions, vol. XLVII, 1751-1752, p. 445-469. 
rapidité certaines de ces œuvres considérables ont été traduites. L'ouvrage principal de François Levaillant (1753-1824), intitulé Voyages de M. Le Vaillant dans l'Intérieur de l'Afrique par le Cap de Bonne Espérance, dans les Années 1780, 81, 82, 83, 84 E 85 (Paris, 1790, 2 vol.), constitue un exemple éclairant. Ce livre fut traduit en anglais par Élizabeth Helme sous le titre Travels from the Cape of Good Hope, into the Interior Parts of Africa, Including many Interesting Anecdotes et publié la même année que la version française. Dans sa préface, Helme souligna qu'elle a supprimé les répétitions de l'auteur. Elle ajoute :

«J'ai également ramolli [...] quelques passages qui pourraient éventuellement être pris pour de simples effusions de fantaisie et de vivacité d'un auteur français $[\ldots] »^{14}$

La censure effectuée par la traductrice porte surtout sur l'ethnographie des populations africaines. En général, les traducteurs insistaient sur le fait qu'ils respectaient scrupuleusement le texte original, soulignant à la fois la valeur scientifique de l'auteur ainsi que celle de leur propre travail.

\section{La prise de pouvoir de l'exploration «banksienne »(1788-1798)}

$\mathrm{Au} \mathrm{XVIII}$ e siècle, les géographes commencèrent à s'intéresser à l'intérieur de l'Afrique, et surtout aux cours des grands fleuves - le Nil, le Niger, le Sénégal, le Gambie. Par manque d'études récentes sur ce thème, ils firent référence à trois autorités. L'accès à leurs œuvres ne fut possible qu'à travers des traductions. La première autorité était le géographe arabe Charif Al Idrissi (c.1100-c.1165), qui avait travaillé à la cour du roi Roger en Sicile. Son livre, intitulé Kitâb Nuzhat al Mushtâq ou Kitab Rudjâr, date de 1154. L'auteur fut le premier à lancer des hypothèses sur la situation géographique du bassin du Nil. En général, les géographes des Lumières utilisaient la traduction latine de Gabriel Sionita (1577-1648), datant de $1619^{15}$. La seconde autorité était Jean Léon l'Africain (c.1494c.1554). En 1526, il publia à Rome une géographie de l'Afrique contenant une description du Niger. Ce livre en latin fut traduit en italien (1550), en français (1556), et en anglais $(1600)^{16}$. La troisième personne à constituer une référence en la matière était le militaire espagnol Luis del Marmol y Carvajal (1520-1600). Sa description de l'intérieur de l'Afrique, publiée entre 1573 et 1599, témoigne que l'auteur avait parcouru le Sahara. En France, on utilisait la traduction de Nicolas Perrot d'Ablancourt (1606-1664), datant de $1667^{17}$.

Le débat sur l'intérieur de l'Afrique était donc alimenté par les traductions de ces trois « autorités ». En même temps, les géographes comprenaient l'importance de lire d'autres auteurs africains ou arabes qui pouvaient donner des informations basées sur des observations directes. En France surtout, le rôle des orientalistes dans le développement de nouvelles connaissances géographiques ne peut pas être sous-estimé. On constate que certaines fonctions officielles de l'État s'impliquèrent graduellement dans le processus de la traduction et de l'interprétation de récits concernant $l^{\prime}$ Afrique ${ }^{18}$. Ici, il faut penser surtout aux Secrétaires-Interprètes du Roi en langues orientales. Il s'agit d'une charge officielle mise à la disposition de la diplomatie française. En ce qui concerne l'étymologie

14. Travels from the Cape of Good Hope, into the Interior Parts of Africa, Including many Interesting Anecdotes... Translated from the French of Monsieur Vaillant, London, Printed for William Lane, 1790, vol. I, p. 8-9.

15. Geographia nubiensis id est accuratissima totius orbis in septem climata divisi descriptio, continens praesertim exactam universae Asiae, \& Africae, rerumq; in iis hac tenus incogitarum explicationem. Recens ex Arabico in Latinum versa a Gabriele Sionita Syriacarum, E Arabicarum literarum Professore, atque Interprete Regio, E Ioanne Hesronita, earundem Regio Interprete, Maronitis, Parisiis, Ex typograhia Hieronymi Blageart, 1619.

16. La traduction française : Description de l'Afrique, tierce partie du monde, Contenant ses Royaumes, Régions, Viles, Cités, Fleuves, Animaux, tant aquatiques, que terrestres: coutumes, loix, religion et façon de faire des habitans, avec pourtraits de leurs habis : ensemble autres choses mémorables, E singulières nouveautés: escrite de nôtre tems par Iean Léon Africain, premièrement en langue Arabesque, puis en Toscane, Ė à présent mise en François, Lyon, Jean Temporal, 1556.

17. L'Afrique de Marmol de la traduction de Nicolas Perrot Sieur d'Ablancourt, Paris, Louis Billaine, 1667.

18. H. Van Hoof, « De l'identité des interprètes au cours des siècles », p. 9-19. 
des noms géographiques africains, on fit appel aux connaissances de Jean-Michel Venture de Paradis (1739-1799) ${ }^{19}$. Celui-ci avait passé la première partie de sa carrière dans le service diplomatique et consulaire en Syrie, en Égypte et au Maghreb. Retourné en France, ce « drogman » poursuivit sa carrière comme linguiste. Ayant accédé à la position de Secrétaire-Interprète pour les langues orientales à la Bibliothèque du Roi, il traduisit de nombreux récits de voyage. Les géographes citèrent également Joseph de Guignes (1721-1800), un orientaliste qui lui aussi devint Secrétaire-Interprète pour les langues orientales. Ses interprétations des observations africaines furent intégrées dans plusieurs mémoires qu'il présenta à l'Académie Royale des Inscriptions et Belles-Lettres. Les travaux rédigés par de Guignes donnent des informations détaillées sur les routes commerciales historiques reliant l'Afrique du Nord au bassin du Niger ${ }^{20}$.

Les contemporains de ces deux traducteurs avaient bien compris que les anciennes écritures arabes conservées dans les collections françaises pouvaient potentiellement permettre d'améliorer et d'approfondir la compréhension de la géographie africaine. Un travail plus systématique de repérage, d'analyse et de traduction fut lancé par l'Académie Royale des Inscriptions et Belles-Lettres. Une nouvelle série vit le jour : les Notices et extraits des manuscrits de la Bibliothèque du Roi. Ainsi, par exemple, le Livre des Perles, écrit par Alfassi aux environs de 1450, fut édité et commenté par Antoine-Isaac Silvestre (1759-1838), un spécialiste reconnu des langues sémitiques ${ }^{21}$. Les contemporains étaient particulièrement fascinés par les passages consacrés au cours du Niger.

Dans la dernière décennie du XVIII ${ }^{\mathrm{e}}$ siècle, les expéditions de l'African Association - une organisation privée fondée à Londres par des hommes riches et influents, dont Joseph Banks (1743-1820), président de la Royal Society - bouleversèrent l'exploration africaine ${ }^{22}$. C'est la période de l'épanouissement de l'intérêt britannique pour la géographie de l'intérieur «inconnu» du continent africain. Dans le contexte tendu de l'époque révolutionnaire, on pouvait s'attendre à des réactions françaises. Le cas le plus connu est sans doute celui du Mémoire sur l'intérieur de l'Afrique rédigé par Jérôme de Lalande (1795), qui appela à une mobilisation des forces du côté français, s'appuyant sur des informations fournies par les voyageurs ${ }^{23}$. Le plaidoyer de Lalande en faveur du développement de nouvelles connaissances stratégiques du terrain africain était basé sur la lecture d'une longue série de récits britanniques traduits en français.

La lecture de la première partie des Proceedings de l'African Association, publiée en 1790, a profondément marqué les intellectuels tant britanniques que français. Plus que jamais les connaissances résultant d'une observation directe de la réalité africaine étaient placées au centre de la recherche. Grâce à l'initiative de Banks, le gouvernement britannique développa une nouvelle approche de l'exploration scientifique en général. Elle fut de plus en plus intégrée dans un cadre économique et stratégique à l'échelle mondiale, ce qui stimula les Français à monter des projets compétitifs. Ainsi l'exploration "scientifique » prit progressivement à partir de ce moment une place de plus en plus grande dans les relations entre les puissances, surtout dans l'océan Pacifique mais également ailleurs.

En ce qui concerne l'Afrique, Banks poussa le gouvernement britannique à établir un lien informel mais rigide avec l'African Association. Cette association monta un programme

19. Voir la Thèse de Sibylle Jauffret-Derville, Jean-Michel Venture de Paradis: drogman et orientaliste 1739-1799. 20. «Observations générales sur le commerce \& les liaisons des peuples de l'intérieur de l'Afrique, soit entre eux, soit avec ceux de Barbarie, de l'Égypte \& de l'Arabie, \&c. Lu a la séance publique de l'académie des Inscriptions \& Belles-Lettres, à Paques dernier, par M. de Guignes », Journal des Savans, juillet 1791, p. 393-401.

21. A.-I. Silvestre de Sacy, «Le livre des Perles, recueillies de l'abrégé de l'histoire des siècles ou Abrégé de l'histoire universelle. Par Schéhabeddin Ahmed almokri alfassi. Manuscrits arabes, 792 \& 769 », Notices et extraits des manuscrits de la Bibliothèque du Roi, Lûs au Comité établi par Sa Majesté dans l'Académie Royale des Inscriptions $\mathcal{E}$ Belles-Lettres, t. II, Paris, Imprimerie Royale, 1789, p. 124-163.

22. R. Hallett, "The European Approach to the Interior of Africa in the Eighteenth Century », p. 191-206; R. Hallett, Records of the African Association, 1788-1831, p. 1-41.

23. J. Lalande, Mémoire sur l'intérieur de l'Afrique, Paris, Imprimerie des Administrations Nationales, An troisième de la République [1794-1795]. 
d'exploration ambitieux et envoya en mission des aventuriers tels que l'Américain John Ledyard (1740-1791), l'Anglais Simon Lucas (c.1766-1799), l'Allemand Friedrich Hornemann (1772-1801), le Suisse Johan Ludwig Burckhardt (1784 -1817) et l'Écossais Mungo Park (1771-1806). Ils exécutèrent leurs instructions d'une manière méthodique. Comme on pouvait s'y attendre, les opérations britanniques en Afrique suscitèrent un vif intérêt du côté français. La dissertation de Lalande démontre clairement qu'il était un lecteur attentif des Proceedings. Ici, il faut souligner que Lalande a lu ce volume avant qu'il soit disponible à Paris, ce qui atteste des excellentes relations existant entre les communautés scientifiques françaises et britanniques ${ }^{24}$. Les chercheurs français espéraient pouvoir bientôt lire les récits de l'Association dans une traduction française.

Les Français commencèrent bientôt à traduire systématiquement les récits publiés par ordre de l'African Association dans leur propre langue. C'est surtout après le succès du voyage de Mungo Park (1795-1797), qui confirma que le Niger coule vers l'Est, que tout le monde fut convaincu des avantages économiques qui attendaient les Européens à l'intérieur de l'Afrique. Le récit de voyage de Mungo Park, intitulé Travels into the interior of Africa, fut publié en 1798. Ce livre devint immédiatement un succès commercial. La même année, on publia à Paris les Voyages et découvertes dans l'intérieur de l'Afrique par le major Houghton et Mungo Park (An VI). Il s'agit d'une traduction combinée de deux récits, l'un du voyage effectué par Houghton en 1790, l'autre des aventures de Park. Le traducteur est Antoine-Jean-Noël Lallemant, secrétaire au Ministère de la Marine et des Colonies. Ce dernier dédia son travail à son patron, le «Citoyen » Etienne-Eustache Bruix (1759-1805), ministre de la Marine et des Colonies. Ceci démontre une fois de plus l'importance politique de l'ouvrage de Park. En outre, le traducteur jugea utile de publier au début de son livre le mémoire de Lalande qui insistait sur la nécessité de donner une impulsion aux activités françaises en Afrique. Le récit de voyage de Friedrich Conrad Hornemann, décédé en Afrique, fut publié en 1802 en allemand et en anglais. La même année suivit une traduction française, augmentée de notes et d'un mémoire par l'orientaliste Louis-Mathieu Langlès (1763-1824), fondateur de l'École des langues orientales vivantes à Paris ${ }^{25}$.

\section{La percée de la science impérialiste (1798-1830)}

La concurrence entre les Français et les Britanniques en Afrique atteignit son paroxysme en 1798 lorsque Napoléon Bonaparte conquit l'Égypte ${ }^{26}$. Ailleurs en Afrique, les tensions devenaient de plus en plus apparentes. Banks et ses amis à Londres comptaient désormais sur les services offerts par les diplomates britanniques et par leurs réseaux d'interprètes. Ce sont surtout les consuls qui transmirent au monde savant en GrandeBretagne des rapports sur la situation politique et économique en Méditerranée et en Afrique du Nord. Dans les premières décennies du XIX ${ }^{\mathrm{e}}$ siècle, on constate une amplification des opérations, des interventions plus directes des gouvernements dans l'organisation de l'exploration, l'imposition des agendas commerciaux de la science (la question du Niger) et enfin la montée des sociétés de géographie (Paris 1821, Londres 1830). C'est la période des grandes expéditions scientifiques à caractère militaire en Égypte, en Algérie, en Afrique occidentale, au Congo.

Les récits de voyage furent entrainés dans une dynamique de guerre. On accéléra une fois de plus le rythme des traductions de récits produits par la nation ennemie afin d'en déduire des connaissances stratégiques. L'exemple le plus frappant est sans aucun doute le récit de voyage publié en 1802 par Silvestre-Meinrad-Xavier Golberry (1742-1822), intitulé Fragments d'un voyage en Afrique, fait pendant les années 1785, 1786 et 1787, dans les contrées occidentales de ce continent, comprises entre le Cap Blanc de Barbarie, par 20 degrés, 47

24. Ibidem, p. 29.

25. Voyage de F. Hornemann dans l'Afrique septentrionale [...], Paris, Dentu, An IX (1803).

26. P. Bret, L'expédition d'Égypte, une entreprise des Lumières 1798-1801. 
minutes, et le Cap de Palmes, par 4 degrés, 30 minutes, latitude boréale (Paris, Treuttel et Würz, 1802). La même année, Francis Blagdon (1778-1819) en fit une traduction " without abridgment ", intitulée Travels in Africa performed during the years 1785, 1786, and 1787, in the western countries of that continent... (London, 1802). Dans l'introduction, Blagdon insistait sur le fait que la traduction de ce livre de plus de 500 pages fut effectuée en vingt jours! Ce dernier, polyglotte, se spécialisa dans la traduction de récits de voyage. En 1802, il lança sa propre collection, intitulée Modern Discoveries, dans laquelle il publia Travels in Egypt de Dominique Vivant Denon (1747-1825) - un savant, artiste et diplomate qui avait accompagné Napoléon en Égypte. Mais c'est surtout la lecture de la traduction du livre de Golberry qui alarma Joseph Banks et ses amis à Londres.

En effet, le livre ne laissait aucun doute à l'égard du schéma impérial que le régime napoléonien voulait mettre en œuvre en Afrique, c'est-à-dire l'occupation complète de la Sénégambie par des troupes françaises. Banks pressa le gouvernement britannique de prendre une position ferme à ce sujet. Le sous-secrétaire d'État aux Colonies, John Sullivan, prit l'initiative de rédiger un mémorandum dans lequel il recommanda au gouvernement de mettre en place des postes britanniques le long du fleuve Gambie ${ }^{27}$. Avec la reprise de la guerre, le gouvernement britannique engagea Mungo Park pour effectuer une deuxième expédition vers l'intérieur de l'Afrique occidentale. L'objectif était de tracer l'ensemble du cours du Niger jusqu'à son estuaire tout en installant le pouvoir militaire britannique le long de la route.

Il n'est donc pas surprenant de voir qu'au cours des années suivantes les Français commandèrent immédiatement des traductions de tous les récits des expéditions militaires britanniques qui suivirent celle de Mungo Park. Cette politique a été poursuivie après Waterloo (récits des expéditions de Tuckey, original en anglais 1818, traduction en français 1818, et de Gray et Dorchard, original en anglais 1825, traduction en français 1826) ${ }^{28}$. Les Français cherchaient la revanche en Afrique. C'est donc dans cette période que les Annales maritimes et coloniales, une revue officielle contenant des actes et des décisions ministérielles sur les affaires maritimes, publia des traductions partielles et annotées de récits britanniques, expliquant les motivations de ces initiatives soi-disant «scientifiques ${ }^{29}$.

Avec la conquête française de l'Algérie en 1830, la valeur informative des récits de voyage fut une fois de plus soulignée. Non seulement publiait-on les anciens récits de voyageurs français comme Peyssonnel et Desfontaines qui déjà au XVIII ${ }^{\mathrm{e}}$ siècle avaient donné des descriptions de l'arrière-pays d'Alger ${ }^{30}$, mais aussi la traduction du récit de l'Anglais Thomas Shaw qui avait résidé en Barbarie entre 1720 et 1732. C'est en 1830 que parut Voyage dans la régence d'Alger ou description géographique, physique, philologique, etc. de cet état, par le Dr. Shaw (Paris, Chez Marlin, 1830).

Il ne s'agit pas d'une simple traduction. L'auteur, Jacques Maccarthy (1785-1835), ajouta au texte de nombreuses augmentations, des notices géographiques, etc. Celui-ci était un passionné de géographie, résidant à Paris où il était membre de la Société de Géographie.

27. R. Hallet, op. cit., p. 213-215.

28. Relation d'une expédition entreprise en 1816, sous les ordres du capitaine J.-K. Tuckey, pour reconnoître le Zaïre, communément appelé le Congo, fleuve de l'Afrique méridionale; suivie Du Journal du professeur Smith, et de quelques Observations générales sur les Habitans, et de l'Histoire naturelle de la partie du royaume de Congo arrosée par le Zaïre. Le tout précédé d'une Introduction expliquant les motifs qui ont déterminé ce Voyage. Ouvrage publié avec permission des Lords de l'Amirauté. Traduit de l'Anglois, par l'auteur de Quinze jours à Londres [A. J. B. Defauconpret], Paris, Librairie de Gide fils, rue Saint-Marc-Feydeau, $\mathrm{n}^{\circ}$ 20, 1818 ; W. Gray et D. Dochard, Voyage dans l'Afrique occidentale : pendant les années 1818, 1819, 1820 et 1821, depuis la rivière Gambie jusqu'au Niger [...], Paris, Avril de Gastel et Ponthieu, 1826.

29. Par exemple: Annales maritimes et coloniales. Recueil des Lois et Ordonnances royales, Réglemens et Décisions ministérielles, Mémoires, Observations particulières, et générallement de tout ce qui peut intéresser la Marine et les Colonies, sous les rapports militaires, administratifs, judiciaires, nautiques, consulaires et commerciaux; publié avec l'approbation de S. Exc. Le Ministre de la Marine et des Colonies, et sous les auspices de S.A.R. l'Amiral de France, 1818, 2, p. 689-712, 781-803.

30. A.J.C.A. Dureau de la Malle, Peyssonnel et Desfontaines. Voyages dans les régences de Tunis et d'Alger, Paris, Librairie de Gide, 1838. 
Il rédigea une collection de récits de voyages, intitulée Choix de voyages dans les quatre parties $d u$ monde (Paris, Librairie nationale et étrangère, 1821-1822). Maccarthy se consacra à la traduction de beaucoup d'autres livres anglais. Ces connaissances géographiques étaient particulièrement intéressantes pour les militaires. C'est pourquoi il fut attaché à la section de statistique du Dépôt de la Guerre.

En 1830, une partie de l'opinion publique, en France comme en Grande-Bretagne, était favorable à une politique expansionniste en Afrique. Ce sentiment était partagé par la majorité des hommes politiques. Par ailleurs, entre puissances rivales, la traduction des récits dits "de voyage " constitua une source d'information de premier ordre à propos des activités déployées par chacun. Ce phénomène s'est encore intensifié dans les années suivantes, entre autres grâce aux contributions des membres des sociétés de géographie.

\section{Résumé}

Cette contribution s'inscrit dans un projet de recherche financé par le Fonds de la Recherche Scientifique-FNRS, qui vise à mettre en rapport l'intérêt accru des savants pour l'Afrique à la fin $d u X^{X V I I I}{ }^{\mathrm{e}}$ siècle et l'intensification de la compétition économique et militaire entre la France et la Grande-Bretagne. Ce projet ambitionne de démontrer comment une connaissance de plus en plus précise du terrain africain a influencé la façon dont les gouvernements des deux pays ont intégré l'expertise coloniale dans une politique scientifique complexe appropriée aux besoins spécifiques des deux États. L'originalité de cette étude consiste en l'approche comparative des «politiques scientifiques » coloniales successives de la France et de la Grande-Bretagne.

Les récits de voyage ont joué évidemment un rôle important dans la collecte d'informations. Les expéditions ont été suivies de près par le public lettré, par les institutions scientifiques mais aussi par les décideurs politiques des deux côtés de la Manche. En plus de nouvelles données scientifiques, les récits contenaient également des informations d'importance économique et stratégique. Les fonctionnaires ont donc soumis les récits à l'analyse critique dès leur parution. On avait intérêt à faire traduire les récits composés par les concurrents. Cette contribution met l'accent sur la traduction des récits de voyage français et britanniques, et sur leurs arrière-plans politiques. 


\section{Bibliographie}

ABBATTISTA Guido, "The literary mill : per una storia editoriale della Universal History (1736-1765) », Studi settecenteschi, vol. II, 1981, p. 91-133.

AbBatTISTA Guido, "The business of Paternoster Row : towards a publishing history of the 'Universal History' », Publishing History, vol. XVII, 1985, p. 5-50.

AвBATTISTA Guido, "The English Universal History: publishing, authorship and historiography in a European project (1736-1790)», Storia della Storiografia, vol. XXXIX, 2001, p. 103-108.

AlBERTAN-COPPOla Sylviane, "Des récits des voyageurs à l'Histoire générale des voyages : la représentation des Africaines », Dix-huitième siècle, n 44, 2012, pp. 165-181.

BOURGUET Marie-Noëlle, «Voyage, collecte, collections. Le catalogue de la nature (fin XVII -début XIX siècles)», in LECOQ Danielle et CHAMBARD Antoine (dir.), Terre à découvrir. Terres à parcourir. Exploration et connaissance du monde XII ${ }^{e}-\mathrm{XIX}^{e}$ siècles, ParisMontréal, L'Harmattan, 1998, p. 185-207.

BRET Patrice, L'expédition d'Égypte, une entreprise des Lumières 1798-1801, Paris, Académie des Sciences, 1999.

CARNEY Judith A. et ROSOMOFF Richard Nicholas, In the Shadow of Slavery. Africa's Botanical Legacy in the Atlantic World, Berkeley, University of California Press, 2009.

CuRRAn Andrew S., "Imaginer l'Afrique au siècle des Lumières », Cromohs, vol. X, 2005, p. 1-14.

CULtRu Prosper, «Les faux d'un historien du Sénégal », La Quinzaine coloniale, janvierdécembre 1910, p. 399-402.

CURRAN Andrew S., The Anatomy of Blackness, Science and Slavery in an Age of Enlightenment, Baltimore, The Johns Hopkins University Press, 2011.

DiOP David, GRAILLE Patrick et ZATORSKA Izabella (dir.), L'Afrique [= numéro thématique de la revue Dix-huitième Siècle, n44, 2012].

GALlouËT Catherine, DiOP David, Boceuillon Michèle et LAHOUATI Gérard (dir.), L'Afrique du Siècle des Lumières: savoirs et représentations, Oxford, Voltaire Foundation, 2009.

HAIR Paul E.H., "The Falls of Félou : A Bibliographical Exploration », History in Africa, vol. XI, 1984, p. 113-130.

HalletT Robin, «The European Approach to the Interior of Africa in the Eighteenth Century ", The Journal of African History, vol. IV, 1963, n 2, p. 191-206.

HALLETT Robin, Records of the African Association, 1788-1831, London, Thomas Nelson, 1964.

Halleux Robert, "Aux origines des politiques scientifiques", Archives internationales d'histoire des sciences, vol. LXII, ${ }^{\circ}$ 169, décembre 2012, p. 439-450.

HILL Matthew H., « Towards a chronology of the publications of Francis Moore's Travels into the inland parts of Africa... », History of Africa, vol. XIX, 1992, p. 353-368. 
INGLEBERT Hervé, Le Monde, l'Histoire. Essai sur les histoires universelles, Paris, PUF, 2014.

JAUFFRET-DerVILLE Sibylle, Jean-Michel Venture de Paradis : drogman et orientaliste 17391799. Thèse de doctorat sous la direction de G. Alleaume, soutenue le 19 avril 2011, Université Aix-Marseille 1 (Espaces, cultures, sociétés).

KenNeDy Dane, The Last Blank Spaces. Exploring Africa and Australia, Cambridge-London, Harvard University Press, 2013.

LAW Robin, « The Original Manuscript Version of William Snelgrave's New Account of Some Parts of Guinea », History in Africa, vol. XVII, 1990, p. 367-372.

MAsson Paul, «Une double énigme: André Brue », Revue d'histoire des colonies, vol. XX, $\mathrm{n}^{\circ} 85,1932$, p. $9-34$.

RICUPERATI Giuseppe, «Universal History : storia di un projetto europea. Impostori, storici ed editori nella Ancient Part », Studi settecenteschi, vol. II, 1981, p. 7-90.

SGARD Jean, «Prévost: de l'ombre aux lumières (1736-1746) », Studies on Voltaire and the Eighteenth Century, n²7, 1963, pp. 1479-1487.

VANDERSMISSEN Jan, «Les voyages organisés par ordre du Roi », Archives internationales d'Histoire des Sciences, vol. LXII, n 169, décembre 2012, p. 491-504.

VAN Hoof Henri, "De l'identité des interprètes au cours des siècles », Hieronymus Complutensis, vol. III, 1996, p. 9-19. 